\title{
Dynamical correlations in the one-dimensional electron gas with short-range interactions
}

\author{
E. Demirel and B. Tanatar ${ }^{\mathrm{a}}$ \\ Department of Physics, Bilkent University, Bilkent, 06533 Ankara, Turkey
}

Received 11 December 1998 and Received in final form 25 April 1999

\begin{abstract}
We study the dynamical correlation effects in a one-dimensional Fermion gas with repulsive delta-function interaction within the quantum version of the self-consistent field approximation of Singwi, Tosi, Land, and Sjölander [Phys. Rev. 176, 589 (1968)]. The dynamic correlation effects are described by a frequency dependent local-field correction $G(q, \omega)$. There is a corresponding local-field factor $J(q, \omega)$ for the spin-density correlations. We investigate the structure factors, spin-dependent pair-correlation functions, the frequency dependences of $G(q, \omega)$ and $J(q, \omega)$, and the plasmon dispersion relation within this formalism. We compare our results with other theoretical approaches, in particular the static version of the self-consistent field approximation to highlight the importance of dynamical correlations.
\end{abstract}

PACS. 71.10.Ca Electron gas, Fermi gas - 71.45.Gm Exchange, correlation, dielectric and magnetic functions, plasmons - 73.20.Dx Electron states in low-dimensional structures (superlattices, quantum well structures and multilayers)

\section{Introduction}

The model of an one-dimensional (1D) electron gas is of continuing interest because of its potential applicability to realistic systems such as semiconductor quantum wires, conducting organic materials, and newly discovered carbon tubules. Inasmuch as the long-range Coulomb forces influence and determine the ground-state and transport properties of these systems, the one-dimensional Fermi gas with a repulsive contact interaction also provides an useful paradigm. There are several reasons. Firstly, the exact solution for the ground-state energy has been given by Yang [1] within the Bethe ansatz approach and numerically calculated by Friesen and Bergersen [2]. Thus, various approximate methods may be checked against the exact results. Secondly, the simple form of the interaction ( $\delta$-function in real space) allows us to introduce approximate schemes which go beyond the mean-field as has been explored by Nagano and Singwi [3,4]. The study of 1D electron gas with various interaction laws in different contexts has been thoroughly discussed in the literature [5].

The correlation effects not fully described by the meanfield random-phase approximation (RPA) are accounted for within the concept of local-field corrections. The selfconsistent field method of Singwi et al. [6]. (STLS) treats the short-range correlation effects in charged quantum systems by taking into account the repulsion hole of each electron. The earlier work of Friesen and Bergersen [2] and the recent studies of Gold $[7,8]$ have shown that

\footnotetext{
a e-mail: tanatar@fen.bilkent.edu.tr
}

the STLS method applied to the 1D electron gas with $\delta$-function interaction yields reasonable agreement with the exact ground-state energy up to intermediate coupling strengths.

In this work we apply the dynamical (or quantum) version of the STLS theory to the problem of 1D electron gas with short-range interaction and investigate the dynamical properties. There are several motivations to study the effects of dynamical correlations. In recent years the dynamical correlation effects have been recognized to play an important role in describing the many-body properties of electronic systems. Richardson and Ashcroft [9] have developed wave vector and frequency dependent local-field factors for a 3D electron gas using a variational approach to solve integral equations for the densitydensity and spin-density response functions. Takayagani and Lipparini [10] have solved the Bethe-Goldstone equation in the particle-hole channel to determine the effective interaction between the electrons. Among the possible occurrences where the dynamical correlations become important are the effective electron-electron interactions relevant for Coulomb interaction induced superconductivity, calculation of the plasmon lifetime, and exchangecorrelation potentials in the context of density-functional theory [11]. The dynamical version of the STLS approximation (qSTLS) has been mainly employed to study the correlation effects in charged quantum liquids interacting via long-range forces [12-17]. The theory takes dynamical nature of the Pauli correlation hole into account and treats the Coulomb correlation hole at a static level $[12,15]$. In the present problem the electrons interact 
with a short-range (contact) interaction and it is of interest to compare and contrast the results of the dynamic STLS theory with the other approaches. We find that there are significant differences in the ground-state correlation functions and the local-field corrections between the static and dynamic STLS methods.

The rest of this paper is organized as follows. In the next section we outline the qSTLS method in application to $1 \mathrm{D}$ electrons with short-range interactions. In Section 3 we present the results of our self-consistent calculations and discuss the differences between the static and qSTLS approximations. We conclude with a brief summary in Section 4 .

\section{Model and theory}

We consider a system of electrons in $1 \mathrm{D}$ interacting via a contact potential $V\left(r_{1}, r_{2}\right)=V_{0} \delta\left(r_{1}-r_{2}\right)$, where $V_{0}$ is the interaction strength. In terms of the electron mass $m$ and the density of the particles $n$, we use the dimensionless parameter $\gamma=m V_{0} / n$ to characterize the strength of the coupling (we take $\hbar=1$ ). The Fermi wave vector $k_{\mathrm{F}}$, is related to the linear density by $n=2 k_{\mathrm{F}} / \pi$. We calculate the correlation effects employing the self-consistent field approximation of Singwi et al. [6]. (STLS) generalized to include dynamic correlations [12-14] which introduces a local-field correction to the bare interaction $V(q)$ arising from the short-range correlations between the electrons.

The derivation leading to the frequency-dependent local-field factor is similar to that in the static STLS approximation. The hierarchy of coupled equations satisfied by the Wigner distribution functions is truncated with the assumption that the two-particle Wigner distribution function may be written as a product of one-particle distribution functions and the pair-correlation function $[12,13]$. The density-density response function without any loss of generality is given by

$$
\chi^{d}(q, \omega)=\frac{\chi_{0}(q, \omega)}{1-V_{\mathrm{eff}}^{\mathrm{s}}(q, \omega) \chi_{0}(q, \omega)},
$$

where $\chi_{0}(q, \omega)$ is the zero-temperature dynamic susceptibility of a non-interacting electron gas [18] evaluated on the imaginary frequency axis

$$
\chi_{0}(q, i \omega)=\frac{m}{2 \pi q} \ln \left|\frac{\omega^{2}+\omega_{-}^{2}}{\omega^{2}+\omega_{+}^{2}}\right|,
$$

in which $\omega_{ \pm}=\left|q^{2} / 2 m \pm q k_{\mathrm{F}} / m\right|$ are the boundaries of particle-hole excitation region. Similarly, the spin-density response function can be written as

$$
\chi^{\mathrm{s}}(q, \omega)=-\frac{\chi_{0}(q, \omega)}{1-V_{\mathrm{eff}}^{\mathrm{a}}(q, \omega) \chi_{0}(q, \omega)} .
$$

In the quantum version of the STLS approximation, $V_{\mathrm{eff}}^{\mathrm{s}}(q, \omega)$ and $V_{\mathrm{eff}}^{\mathrm{a}}(q, \omega)$ are, respectively, the spinsymmetric and spin-antisymmetric effective dynamic potentials. They are expressed as

$$
V_{\mathrm{eff}}^{\mathrm{s}}(q, \omega)=V(q)[1-G(q, \omega)]
$$

and

$$
V_{\mathrm{eff}}^{\mathrm{a}}(q, \omega)=V(q) J(q, \omega),
$$

where $G(q, \omega)$ and $J(q, \omega)$ are the spin-symmetric and spin-antisymmetric dynamic local-field factors given by $[12,13,15]$

$$
G(q, \omega)=-\frac{1}{n} \int_{-\infty}^{\infty} \frac{\mathrm{d} k}{2 \pi} \frac{\chi_{0}(q, k ; \omega)}{\chi_{0}(q, \omega)} \frac{V(k)}{V(q)}[S(q-k)-1],
$$

and

$$
J(q, \omega)=\frac{1}{n} \int_{-\infty}^{\infty} \frac{\mathrm{d} k}{2 \pi} \frac{\chi_{0}(q, k ; \omega)}{\chi_{0}(q, \omega)} \frac{V(k)}{V(q)}[\tilde{S}(q-k)-1] .
$$

In the above expressions the inhomogeneous free-electron response function

$$
\chi_{0}(q, k ; \omega)=2 \int_{-\infty}^{\infty} \frac{\mathrm{d} p}{2 \pi} \frac{f(p+k / 2)-f(p-k / 2)}{\omega-p q / m+\mathrm{i} \eta},
$$

has been used, with $f(q)$ being the distribution function for non-interacting electrons. For $k=q$, the inhomogeneous response function reduces to the familiar homogeneous case $\chi_{0}(q, \omega) . S(q)$ and $\tilde{S}(q)$ are the static density and spin-density structure factors. They are related to the $\chi^{\mathrm{d}}(q, \omega)$ and $\chi^{\mathrm{s}}(q, \omega)$ through the fluctuation-dissipation theorem as

$$
S(q)=-\frac{1}{n \pi} \int_{0}^{\infty} \mathrm{d} \omega \chi^{\mathrm{d}}(q, \mathrm{i} \omega)
$$

and

$$
\tilde{S}(q)=\frac{1}{n \pi} \int_{0}^{\infty} \mathrm{d} \omega \chi^{\mathrm{s}}(q, \mathrm{i} \omega),
$$

where we have used the analytic continuation of the response function to the complex frequency plane followed by the Wick rotation of the frequency integral. Using the definition (Eq. (8)) of $\chi_{0}(q, k ; \omega)$ and calculating it on the imaginary frequency axis, we obtain

$$
\chi_{0}(q, k ; \mathrm{i} \omega)=\frac{m}{2 \pi q} \ln \left(\frac{\left(\omega^{2}+\eta^{2}+\omega_{q k-}^{2}\right)^{2}-4 \omega^{2} \eta^{2}}{\left(\omega^{2}+\eta^{2}+\omega_{q k+}^{2}\right)^{2}-4 \omega^{2} \eta^{2}}\right),
$$

where $\omega_{q k \pm}=\left|q k / 2 m \pm q k_{\mathrm{F}} / m\right|$. We have introduced $\eta$ as a small positive quantity to avoid unphysical divergences. These coupled set of equations are solved by iterating between $G(q, \mathrm{i} \omega)$ and $S(q)$, which uses $\chi^{\mathrm{d}}(q, \omega)$ and in turn $G(q, \mathrm{i} \omega)$, until self-consistency is achieved. Similarly, we iterate between $J(q, \omega)$ and $\tilde{S}(q)$, which uses $\chi^{\mathrm{s}}(q, \mathrm{i} \omega)$ and in turn $J(q, i \omega)$, until self- consistency is attained.

\section{Results and discussion}

We first discuss the static density and spin-density structure factors resulting from our self-consistent calculations. 

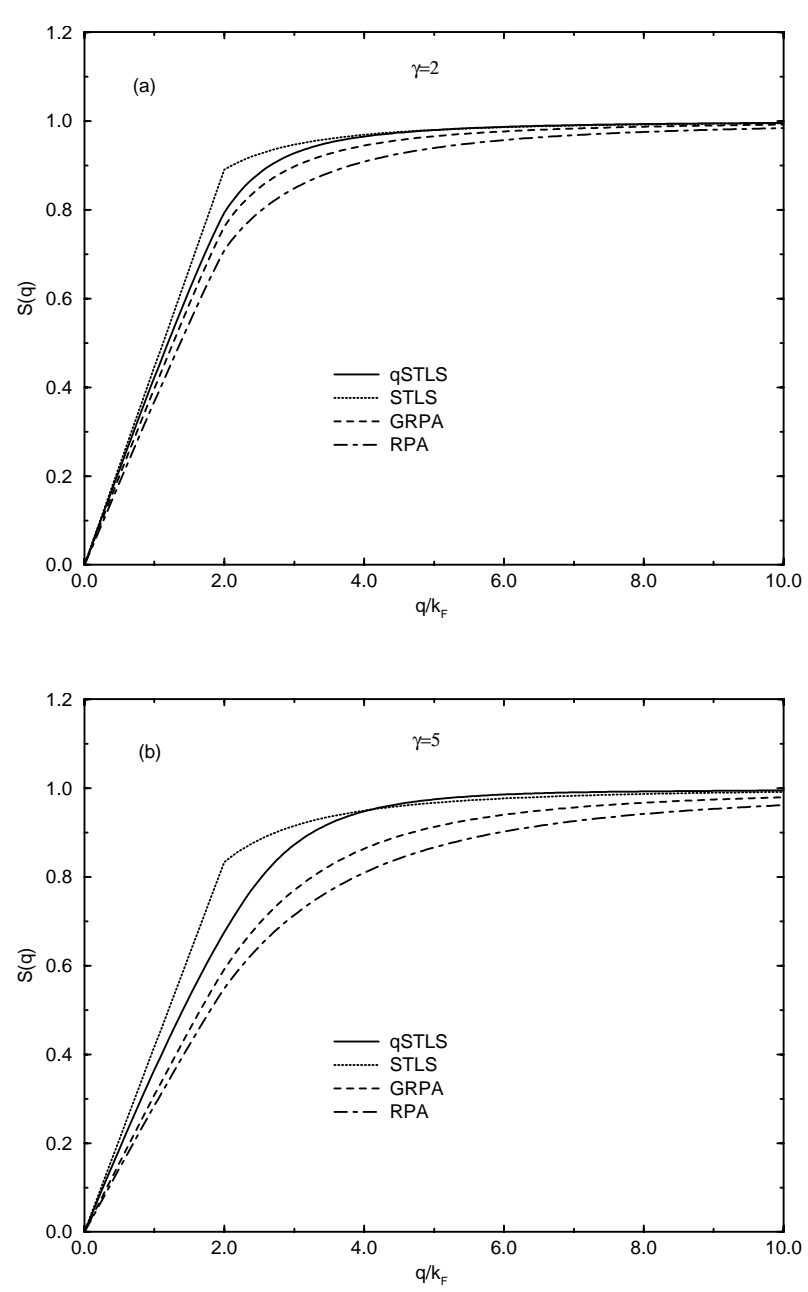

Fig. 1. The static structure factor $S(q)$ within the qSTLS (solid), static STLS (dotted), GRPA (dashed), and RPA (dotdashed) at (a) $\gamma=2$ and (b) $\gamma=5$.

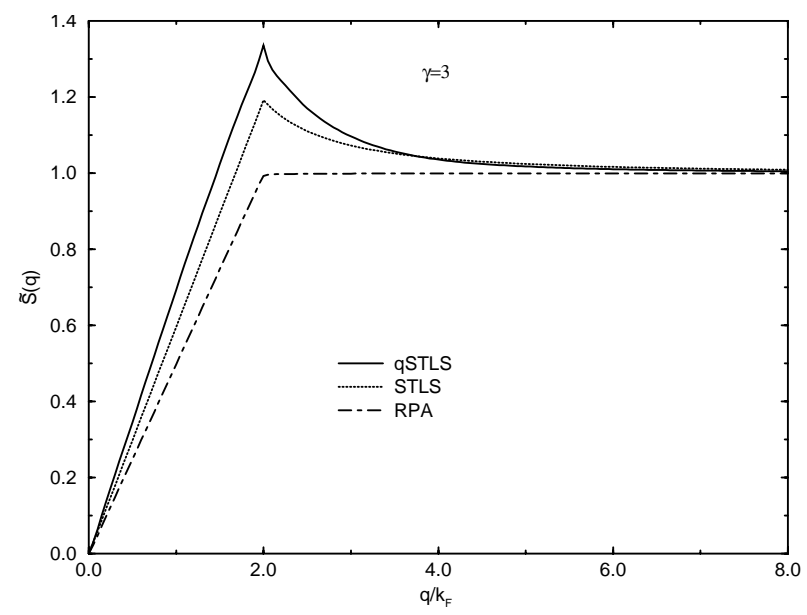

Fig. 2. The static spin-density structure factor $\tilde{S}(q)$ within the qSTLS (solid), static STLS (dotted), and RPA (dot-dashed) at $\gamma=3$.
The calculated results for $S(q)$ are plotted in Figure 1, for two values of $\gamma$. We note that $S(q)$ in the static STLS [7] and the dynamic STLS approximations mainly differ in the region around $q \sim 2 k_{\mathrm{F}}$. There are sizable differences between the RPA and the qSTLS results even for small coupling strengths. The results for the spin-density structure factor $\tilde{S}(q)$ are shown in Figure 2. We observe that a peak structure at $q=2 k_{\mathrm{F}}$ appears in $\tilde{S}(q)$ which is slightly more pronounced in the qSTLS approximation than the static STLS. These differences are attributed to the dynamical correlation effects accounted for in the qSTLS approximation. The RPA without the local-field correction for the spin correlations is tantamount to the non-interacting case, thus do not show such a peak at $q=2 k_{\mathrm{F}}$. We have used our self-consistently determined static structure factor $S(q)$ to calculate the ground-state energy by means of a coupling constant integration. The ground-state energy evaluated in this manner is similar to that calculated within the static STLS approximation $[2,7]$ in the range $0<\gamma<10$. The generalized randomphase approximation (GRPA) of Nagano and Singwi [3] is based on the perturbation theory which sums exactly a large class of diagrams. However, GRPA results in a less satisfactory agreement with the exact ground-state energy.

The probability of finding a particle at a distance $r$ away from a particle situated at the origin is described by the spin-symmetric pair-correlation function $g(r)$ which is the Fourier transform of $S(q)$. In one-dimension the $g(r)$ can be written in the form

$$
g(r)=1-\frac{1}{n \pi} \int_{0}^{\infty} \mathrm{d} k \cos (k r)[1-S(k)] .
$$

Similarly, the spin-antisymmetric pair-correlation function $g_{\mathrm{a}}(r)$ is expressed as

$$
g_{\mathrm{a}}(r)=\frac{1}{n \pi} \int_{0}^{\infty} \mathrm{d} k \cos (k r)[1-\tilde{S}(k)] .
$$

We show the pair-correlation functions $g(r)$ for $\gamma=10$ in different theories in Figure 3. It has been shown by Gold [7] that the pair-correlation function at zero separation $g(0)$, remains positive for the entire range of coupling strengths in the static STLS approximation. The qSTLS theory also yields a positive $g(0)$ for relevant values of $\gamma$, but eventually becomes negative for $\gamma \gtrsim 15$. We note that the GRPA performs less satisfactorily than the qSTLS. Since the range of validity of the STLS (or qSTLS) approximation is limited to the intermediate coupling regime this does not pose a serious practical problem for further applications. From $g(r)$ and $g_{\mathrm{a}}(r)$ we can calculate the spindependent electron pair-correlation functions $g_{\uparrow \uparrow}(r)$ and $g_{\uparrow \downarrow}(r)$ as

$g_{\uparrow \uparrow}(r)=g(r)-g_{\mathrm{a}}(r), \quad$ and $\quad g_{\uparrow \downarrow}(r)=g(r)+g_{\mathrm{a}}(r)$

$g_{\uparrow \uparrow}(r)$ and $g_{\uparrow \downarrow}(r)$ describe the parallel and anti-parallel spin correlations, respectively. The results obtained for $g_{\uparrow \uparrow}(r)$ and $g_{\uparrow \downarrow}(r)$ are shown in Figure 4 for various values of $\gamma$ in different approximations. We find that $g_{\uparrow \uparrow}(r)$ 


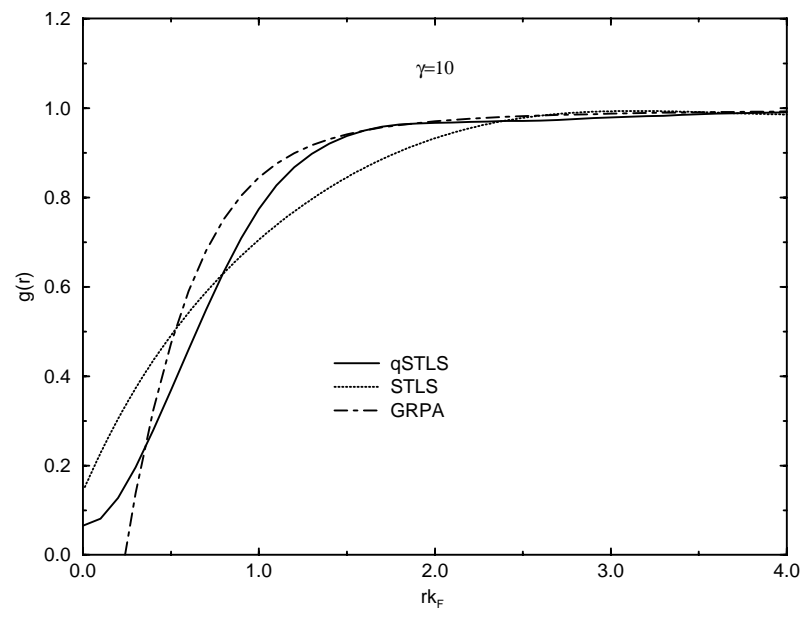

Fig. 3. The pair-correlation function $g(r)$ within the qSTLS (solid), static STLS (dotted), and GRPA (dot-dashed) at $\gamma=$ 10.

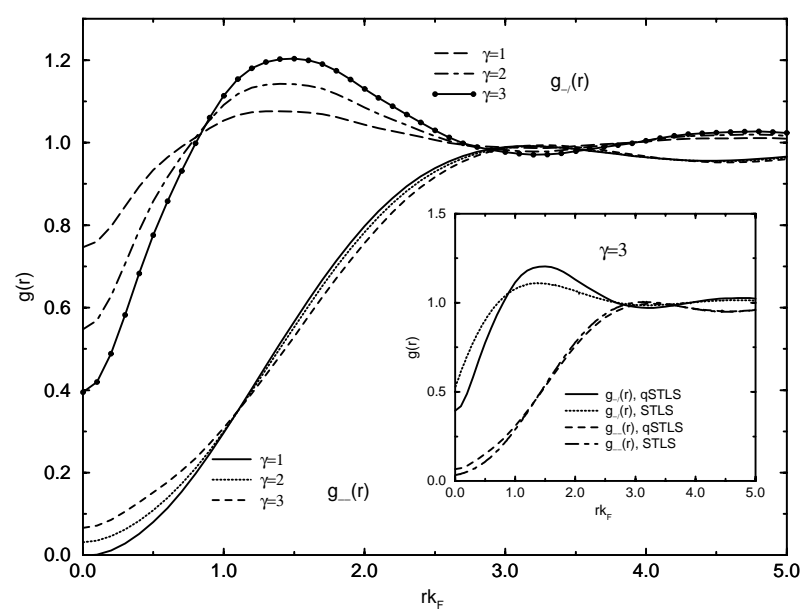

Fig. 4. The spin-dependent pair-correlation functions $g_{\uparrow \uparrow}(r)$ and $g_{\uparrow \downarrow}(r)$ obtained from $g(r)$ and $g_{\mathrm{a}}(r)$ within the qSTLS for different $\gamma$ values. The inset compares the qSTLS and static STLS approximation results at $\gamma=3$.

remains roughly the same for all $\gamma$ values with very little variation at $g_{\uparrow \uparrow}(0) . g_{\uparrow \downarrow}(r)$, on the other hand, shows greater variance as $\gamma$ changes. In the inset of Figure 4 we compare the results of qSTLS and static STLS approaches and find more structure in $g_{\uparrow \downarrow}(r)$ within the qSTLS approximation which shows the effects of dynamic correlations. The differences between the static and qSTLS predictions for the pair-correlation function stem from the dynamical treatment of the Pauli correlation hole in the latter approach.

From our self-consistent calculations of the correlation effects we obtain the spin-symmetric $G(q, \mathrm{i} \omega)$, and spinantisymmetric $J(q, i \omega)$, dynamic local-field factors on the imaginary frequency axis. Similar to the situation in electron gas problems with long-range Coulomb interaction $G(q, \mathrm{i} \omega)$ and $J(q, \mathrm{i} \omega)$ are rather smooth and uninteresting functions of $\mathrm{i} \omega$. The real and imaginary parts of $G(q, \omega)$ and $J(q, \omega)$ may be obtained from $G(q, i \omega)$ and $J(q, i \omega)$

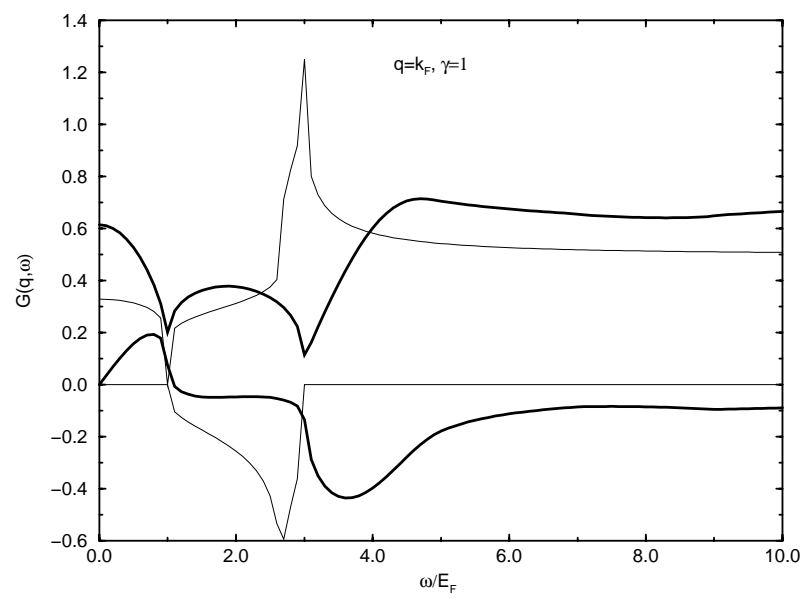

Fig. 5. The real (top curves) and imaginary (bottom curves) parts of the spin-symmetric local-field factor $G(q, \omega)$ in the qSTLS (thick lines) and the GRPA (thin lines) at $\gamma=1$.

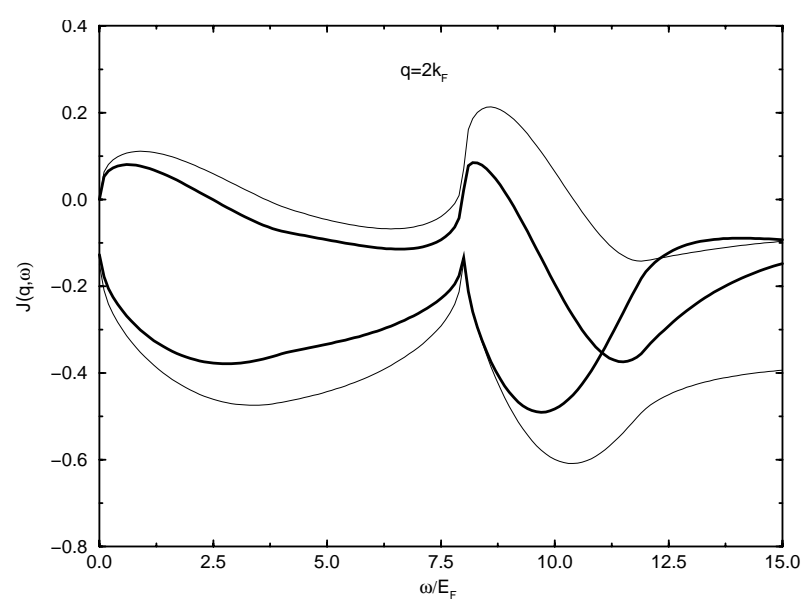

Fig. 6. The real (bottom curves) and imaginary (top curves) parts of the spin local-field factor $J(q, \omega)$ for $\gamma=1$ (thin lines) and $\gamma=3$ (thick lines) in the qSTLS approximation.

by the analytic continuation $\mathrm{i} \omega \rightarrow \omega+\mathrm{i} \eta$ in equations $(6,7)$. We show in Figures 5 and 6 the real and imaginary parts of $G(q, \omega)$ and $J(q, \omega)$, respectively, as functions of the real frequency $\omega$. In Figure 5 we compare the results of qSTLS and the dynamic theory of Nagano and Singwi [3] for $G(q, \omega)$ at $\gamma=1$ and $q=k_{\mathrm{F}}$. There are certain similarities but also marked differences both in the real and imaginary parts. We depict the frequency dependence of $J(q, \omega)$ within the qSTLS approach in Figure 6 for two values of $\gamma$. The results in their oscillatory behavior resembles the case of two-dimensional electron gas [15]. Since the effective electron-electron interaction within the present theory is defined as $V_{0}[1-G(q, \omega)]$, there appears to be a possibility to have attractive interactions for a range of $\omega$ values. It would be interesting to pursue this line of thought for possible superconducting fluctuations in this system. 


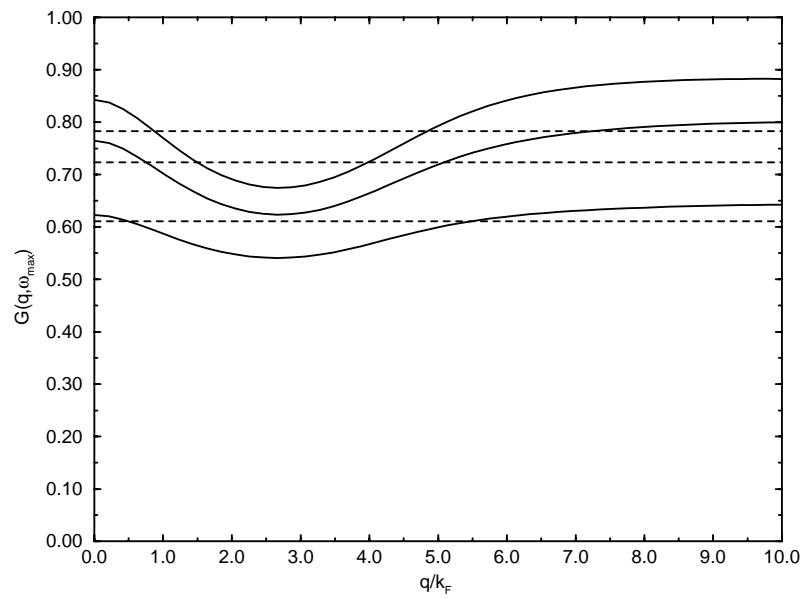

Fig. 7. The large frequency limit of the dynamic local-field factor $G(q, \infty)$ (solid lines) and the $G(q)$ of the static STLS approximation (dashed lines). Curves from bottom to top indicate $\gamma=1,3$ and 5 respectively.

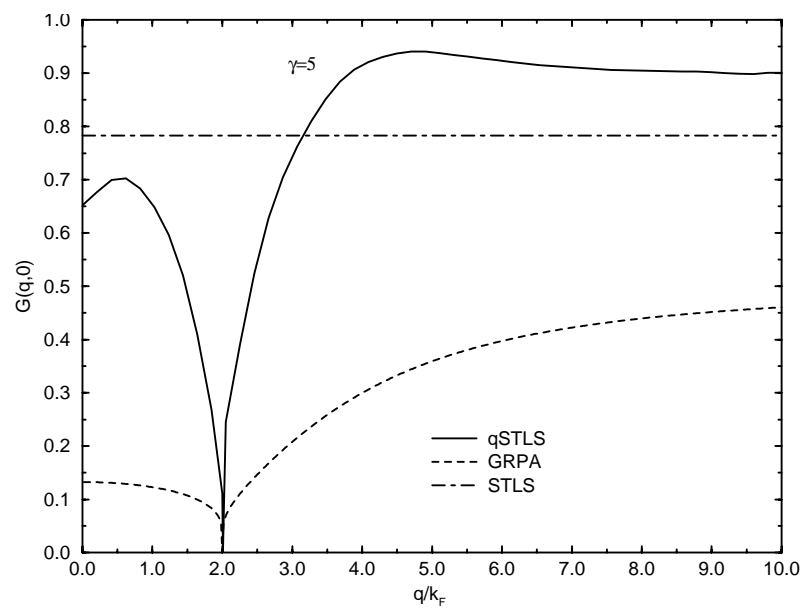

Fig. 8. The zero-frequency local-field factor $G(q, 0)$ calculated within the qSTLS (solid), GRPA (dashed) and static STLS approximation (dot-dashed).

One can show that for fixed $q$ and large $\omega$, the localfield factors behave as

$$
\lim _{\omega \rightarrow \infty} G(q, \omega)=G_{\text {STLS }}(q)+\mathcal{O}\left(\frac{1}{\omega^{2}}\right),
$$

and a similar relation holds for $J(q, \omega)$. Thus, $\operatorname{Im} G(q, \omega)$ vanishes and $\operatorname{Re} G(q, \omega)$ tends to the value given by the static STLS approximation for large $\omega$, as illustrated in Figure 7. The large frequency approximation $G(q, \infty)$ show an interesting dip around $q \approx 2.5 k_{\mathrm{F}}$ which is absent in the static STLS. We actually plot $G\left(q, \omega_{\max }\right)$ at some large frequency value (but not infinite), thus the large $q$ limit does not exactly coincide with $G(q)$ of the static STLS calculation [8]. The near similarity of the constant local-field factor within the static STLS and highfrequency limit of $G(q, \omega)$ within the qSTLS theories, give further credence to the Friesen and Bergersen [2] and Gold's [7] results of ground-state energy.
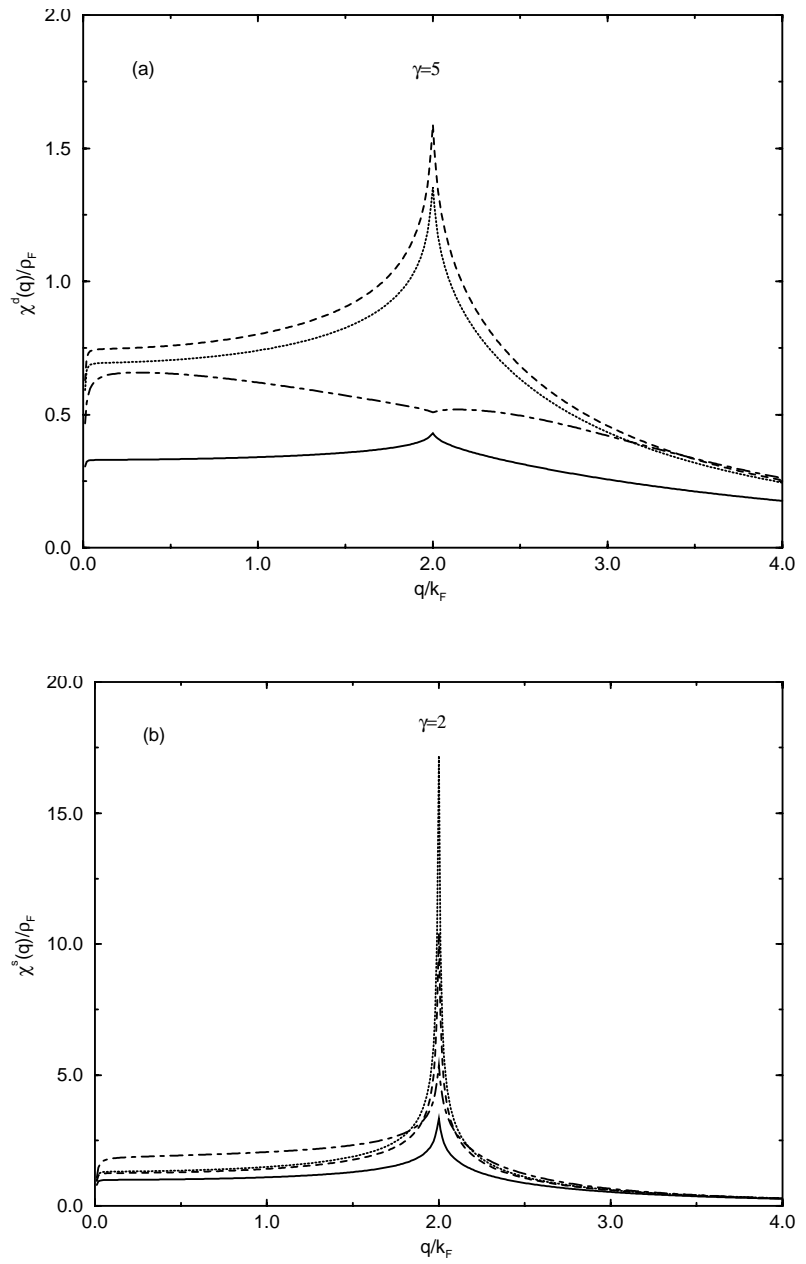

Fig. 9. (a) The static susceptibility $\chi^{\mathrm{d}}(q)$ at $\gamma=5$ in the RPA (solid), static STLS (dotted), and qSTLS with $G(q, \infty)$ (dashed) and $G(q, 0)$ (dot-dashed). (b) The same for the static spin susceptibility $\chi^{\mathrm{s}}(q)$ at $\gamma=2$.

The zero-frequency limit of the spin-symmetric localfield factor $G(q, 0)$, is of interest in some practical applications. In Figure 8 we show the results for $G(q, 0)$ in qSTLS and Nagano and Singwi's dynamic theory [3] as well as the local-field factor in the static STLS approximation at $\gamma=5$. We note that $G(q, 0)$ has the peculiar behavior at $q=2 k_{\mathrm{F}}$. One can analytically show using equation (6) that $G\left(2 k_{\mathrm{F}}, 0\right)$ vanishes, and our numerical calculations confirm this. The dynamical theory of Nagano and Singwi [3] also predicts the same behavior, whereas the static STLS [7] approach with a $q$-independent local-field factor completely misses it. Since a similar behavior of $G(q, 0)$ has been found in quasi-one-dimensional electron gas with long-range interactions [17], we conclude that it is the property of $1 \mathrm{D}$ systems in general coming from dynamical considerations, irrespective of the interaction type. However, there are significant differences between the GRPA and the qSTLS results for $G(q, 0)$ as Figure 8 illustrates. To show the effect of $G(q, 0)$ in relation 

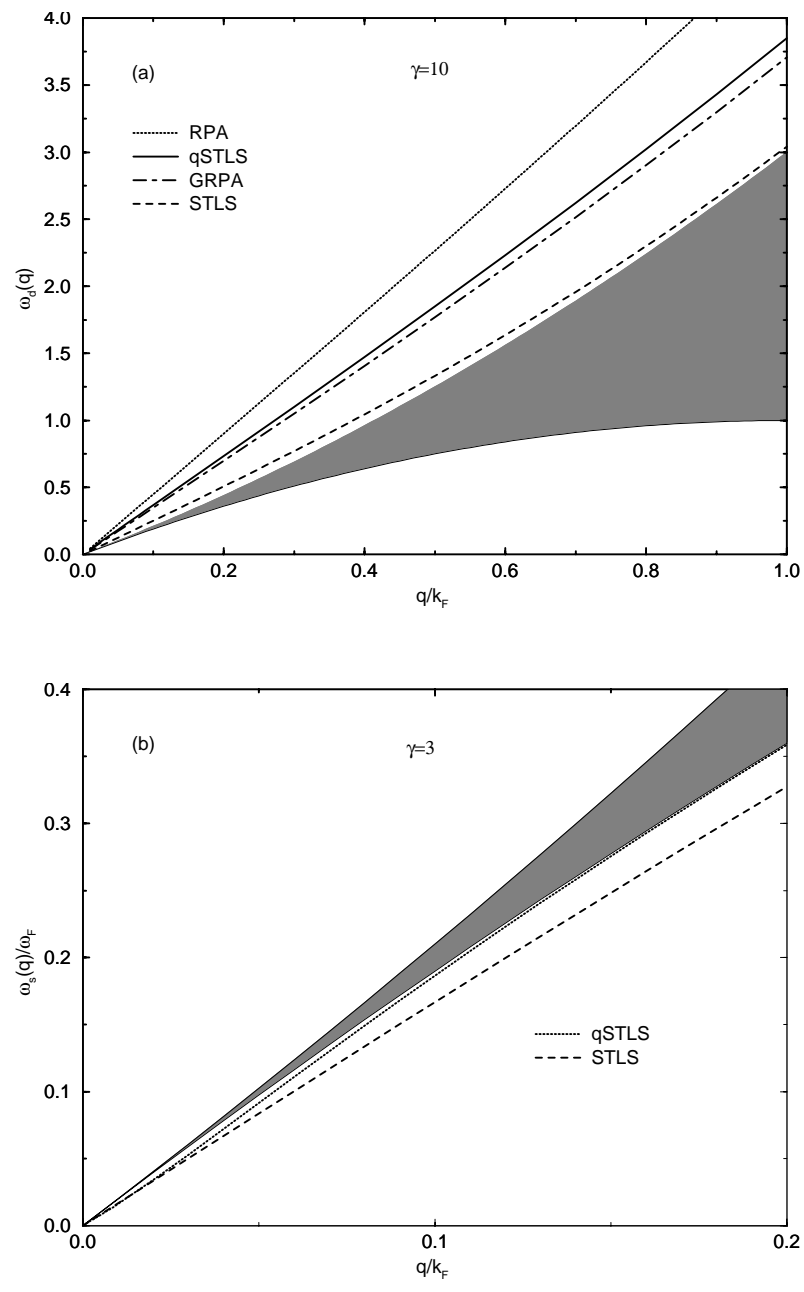

Fig. 10. (a) The dispersion of the collective modes for density fluctuations $\omega_{\mathrm{d}}(q)$ in different theories at $\gamma=10$. The solid, dashed, dot-dashed, and dotted lines are for the qSTLS approximation, the static STLS approximation, GRPA, and RPA, respectively. (b) The same for collective spin-density modes at $\gamma=3$. The dotted and dashed lines indicate qSTLS and static STLS approximations, respectively. The shaded regions in both cases indicate the particle-hole excitation continuum.

to the static $G(q)$ and the high frequency limit $G(q, \infty)$, we plot the static density and spin susceptibilities $\chi^{\mathrm{d}}(q)$ and $\chi^{\mathrm{s}}(q)$, respectively, in Figure 9. The static susceptibilities are calculated by taking the zero-frequency limit of equations $(1,3)$. We find that the STLS and qSTLS with $G(q, \infty)$ results are very near as equation(15) suggests. However, $\chi^{\mathrm{d}}(q)$ calculated with $G(q, 0)$ lies somewhere between the above approximations and the RPA, showing a less pronounced peak at $q=2 k_{\mathrm{F}}$. It has been argued [12] that when $G(q, 0)$ exceeds unity, the systems becomes unstable towards the formation of a charge density wave (CDW). We find no evidence for a CDW instability for the range of coupling strengths $(0<\gamma \lesssim 15)$ we investigate. Qualitatively similar conclusions may be drawn for the static spin susceptibility $\chi^{\mathrm{s}}(q)$ as shown in Figure $9 \mathrm{~b}$. These predictions for the static response functions as well as other ground-state properties may in principle be tested by Quantum Monte-Carlo methods [19] when they are available.

Using the fluctuation dissipation theorem $S(q, \omega)=$ $-1 /(n \pi) \operatorname{Im} \chi(q, \omega)$, we have also calculated the dynamic structure factor $S(q, \omega)$ within the present approach. The dynamic structure factor resembles that calculated by Nagano and Singwi $[3,4]$ in their GRPA with some differences. Most noteworthy of these is that the $S(q, \omega)$ becomes slightly negative for $\omega>\omega_{+}$(the upper edge of the particle-hole continuum), indicating the violation of causality. Although for small values of $\gamma$ the negative part in $S(q, \omega)$ is barely noticeable, it becomes serious for large coupling strengths. This is a common shortcoming of the qSTLS theory as seen in various other applications $[13,15]$. In the case of the dynamic spin structure factor $\tilde{S}(q, \omega)$, the results of qSTLS are rather similar to that calculated within the static STLS approximation.

Finally, we consider the collective excitations in the system. The plasmon excitation spectrum can be obtained from the poles of the response function. The dispersion of the collective modes for density fluctuations $\omega_{\mathrm{d}}(q)$ are obtained by solving the equation

$$
1-V_{0}\left[1-G\left(q, \omega_{\mathrm{d}}\right)\right] \chi_{0}\left(q, \omega_{\mathrm{d}}\right)=0 .
$$

In the RPA and static STLS approximation the localfield correction does not depend on $q$ and $\omega$. Therefore, within these approaches, equating the denominator of the response functions to zero we can obtain the analytic form of the dispersion of the collective modes for density fluctuations $\omega_{\mathrm{d}}(q)$ as

$$
\frac{\omega_{\mathrm{d}}(q)}{E_{\mathrm{F}}}=\left[\frac{\omega_{+}^{2} A_{\mathrm{d}}(q)-\omega_{-}^{2}}{A_{\mathrm{d}}(q)-1}\right]^{1 / 2},
$$

where

$$
A_{\mathrm{d}}(q)=\exp \left[\pi^{2} q /\left(2 k_{\mathrm{F}} \gamma[1-G(\gamma)]\right)\right] .
$$

In the qSTLS and GRPA of Nagano and Singwi [3], the local-field corrections are complex and frequency dependent. In these cases we cannot solve equation (16) analytically for $\omega_{\mathrm{d}}(q)$. We have solved this equation for qSTLS and GRPA numerically and compared our results with the RPA and static STLS approximations. In Figure 10a we show $\omega_{\mathrm{d}}(q)$ at $\gamma=10$ in various approximations. The RPA without the local-field corrections yields a well-defined plasmon dispersion which is largely above the particlehole excitation region. In the static STLS approximation, on the other hand, the plasmons are suppressed and $\omega_{\mathrm{d}}(q)$ stays very close to $\omega_{+}$. We observe that the approximation schemes which take the dynamical correlations into account, namely the qSTLS approach and GRPA of Nagano and Singwi [3] produce plasmon dispersion somewhere between the RPA and static STLS. This result is interesting since the qSTLS theory as applied to long-range Coulomb problems $[2,6,15]$ typically predicts plasmon curves below the static STLS results. As the experimentally measured [20] plasmon dispersions in quasi-one-dimensional 
electron systems are close to the RPA curve, we believe that our results may have some relevance [21]. Das Sarma and Hwang [21] argue that because experimental results are readily explained by the RPA, local-field corrections do not exist in 1D electron systems. Our calculations indicate that if dynamical correlations are taken into account, it would be possible to preserve the notion of local-field corrections. For small values of $\gamma$ the plasmon dispersion curves in all approximations are very close to each other, but we have found similar behavior of $\omega_{\mathrm{d}}(q)$ for other values of $\gamma$ we have examined. In a similar vein, we also calculate the collective spin excitations $\omega_{\mathrm{s}}(q)$ by solving

$$
1-V_{0} J\left(q, \omega_{\mathrm{s}}\right) \chi_{0}\left(q, \omega_{\mathrm{s}}\right)=0 .
$$

Figure 10b shows the dispersion of collective spin-density modes within the static STLS and present dynamical qSTLS approximations for the coupling strength $\gamma=3$. We find that $\omega_{\mathrm{s}}(q)$ within the dynamical approach is much closer to the lower edge of the particle-hole continuum than the results of static STLS. This seems to agree with the experimental observation of intrasubband spin-density excitations by Schüller et al. [22].

In view of the above results, we compare and contrast the some of the main features of static and qSTLS approaches. Both approximation schemes provide a reasonable estimate for the ground-state energy and therefore pressure and compressibility in comparison with the exact results [1-3]. There are noteworthy differences between the predictions of the two approaches when the ground-state correlation functions are considered. The dynamical treatment of the correlation effects described by $G(q, \omega)$ and $J(q, \omega)$ indicate more structure. The static susceptibility function $\chi^{\mathrm{d}}(q)$ reflects the significance of using inherently frequency dependent $G(q, \omega \rightarrow 0)$. The wave vector and frequency dependent local-fields are also expected to introduce marked differences in quantities like effective interactions, and quasi-particle properties (self-energy, etc.) of the 1D electron gas. We note, however, that the dynamical correlations included in the present scheme are only approximate (i.e. only the Pauli correlation hole is taken into account), thus various deficiencies may be attributed to this incompleteness.

\section{Summary}

In summary, we have studied the dynamical correlation effects in a 1D electron gas with short-range interaction within the dynamical STLS theory. The wave vector and frequency dependent local field corrections are useful concepts embodying these correlation effects. We have found that the dynamical correlations give rise to a number of different predictions in the various physical quantities of interest. The static quantities such as the static structure factor and pair-correlation function and dynamic quantities like the plasmon dispersion reflect the importance of dynamical correlations. In particular, the plasmon dispersion is not as heavily suppressed as in the static STLS approach. The dynamical correlations tend to produce more structure in the ground-state correlation functions. As the study of one-dimensional electron gas problems including exchange-correlation effects is gaining more and more attention in recent years our calculations emphasizing the dynamical correlation effects should provide a complementary viewpoint.

This work was partially supported by the Scientific and Technical Research Council of Turkey (TUBITAK) under Grant No. TBAG-1662. We thank Prof. G. Senatore for useful discussions.

\section{References}

1. C.N. Yang, Phys. Rev. Lett. 19, 1312 (1967).

2. W.I. Friesen, B. Bergersen, J. Phys. C 13, 6627 (1980).

3. S. Nagano, K.S. Singwi, Phys. Rev. B 27, 6732 (1983).

4. S. Nagano, K.S. Singwi, S. Ohnishi, Phys. Rev. B 29, 1209 (1984).

5. D.C. Mattis, The Many-Body Problem (World Scientific, Singapore, 1993).

6. K.S. Singwi, M.P. Tosi, R.H. Land, A. Sjölander, Phys. Rev. 176, 589 (1968); K.S. Singwi, M.P. Tosi, Solid State Phys. 36, 177 (1981).

7. A. Gold, Phys. Rev. B 55, 9470 (1997).

8. A. Gold, J. Phys.- Cond. Matter 9, 6877 (1997); ibid. 10, 3547 (1998).

9. C.F. Richardson, N.W. Ashcroft, Phys. Rev. B 50, 8170 (1994).

10. K. Takayanagi, E. Lipparini, Phys. Rev. B 52, 1738 (1995).

11. J.F. Dobson, M.J. Bünner, E.K.U. Gross, Phys. Rev. Lett. 79, 1905 (1997); S. Conti, R. Nifosì, M.P. Tosi, J. Phys.Cond. Matter 9, L475 (1997).

12. T. Hasegawa, M. Shimuzu, J. Phys. Soc. Jap. 38, 1975; K.S. Singwi, in Recent Progress in Many-Body Theories, edited by H. Kümmel, M.L. Ristig (Springer-Verlag, Berlin, 1984).

13. A. Holas, S. Rahman, Phys. Rev. B 35, 2720 (1987).

14. K.H. Schweng, H.M. Böhm, Phys. Rev. B 48, 2037 (1993).

15. R.K. Moudgil, P.K. Ahluwalia, K.N. Pathak, Phys. Rev. B 52, 11945 (1995).

16. K. Tankeshwar, B. Tanatar, M.P. Tosi, Phys. Rev. B 57, 8854 (1998).

17. C. Bulutay, B. Tanatar, Europhys. Lett. 43, 572 (1998).

18. P.F. Williams, A.N. Bloch, Phys. Rev. B 10, 1097 (1974).

19. G. Senatore, S. Moroni, D.M. Ceperley, in Quantum Monte-Carlo Methods in Physics and Chemistry, edited by M.P. Nightingale, C.J. Umrigar (Kluwer, Dordrecht, 1999).

20. A.R. Gõni, A. Pinczuk, J.S. Weiner, J.M. Calleja, B.S. Dennis, L.N. Pfeiffer, K.W. West, Phys. Rev. Lett. 67, 3298 (1991).

21. S. Das Sarma, E.H. Hwang, Phys. Rev. B 54, 1936 (1996).

22. C. Schüller, G. Biese, K. Keller, C. Steinebach, D. Heitmann, P. Grambow, K. Eberl, Phys. Rev. B 54, 17304 (1996). 